\title{
Inhibitory Effect of Human Natural Yeast Killer Toxin-like Candidacidal Antibodies on Pneumocystis carinii
}

\author{
Nathalie Séguy, ${ }^{1}$ Jean-Charles Cailliez, ${ }^{1,2}$ Philippe Delcourt, ${ }^{1}$ \\ Stefania Conti, ${ }^{3}$ Daniel Camus, ${ }^{1,4}$ Eduardo Dei-Cas, ${ }^{4,5}$ \\ and Luciano Polonelli ${ }^{3}$ \\ ${ }^{1}$ Unité 42 INSERM, Villeneuve d'Ascq, France \\ ${ }^{2}$ Faculté Libre des Sciences, Université Catholique de Lille, Lille, France \\ ${ }^{3}$ Istituto di Microbiologia, Università degli Studi di Parma, Parma, Italy \\ ${ }^{4}$ Laboratoire de Parasitologie, Centre Hospitalier Régional Universitaire, \\ Lille, France \\ ${ }^{5}$ Institut Pasteur de Lille, Lille, France
}

\begin{abstract}
Background: Human natural antibodies have been found that owe their candidacidal action to the mimicry of a yeast killer toxin produced by the yeast Pichia anomala (PaKT). Candidacidal human natural antibodies (KTAb) are elicited by and bind to a KT receptor ( $P a \mathrm{KTR}$ ) present on the cell surface of infectious PaKT-sensitive microorganisms. Because of the recognized susceptibility of Pneumocystis carinii organisms to PaKT upon the occurrence of specific PaKTR, we examined whether human natural KTAb could also bind to and inhibit $P$. carinii. Materials and Methods: Immunoaffinity-purified $\mathrm{KTAb}$ from the vaginal fluid of patients affected by candidiasis were tested and compared with $P a K T$ for their ability to inhibit rat-derived $P$. carinii attachment to epithelial lung cells as well as infectivity to nude rats. Immunofluorescence studies were also performed by biotinylated $P a \mathrm{KT}$ in competition with human KTAb to establish their specific binding to PaKTR on the surface of rat-derived and human $P$. carinii organisms.
\end{abstract}

Results: Human natural candidacidal KTAb exerted a strong, specific inhibitory activity against rat-derived $P$. carinii organisms that are susceptible to PaKT itself. The antimicrobial activity of human KTAb was abolished by adsorption with a specific PaKT-neutralizing mAb KT4. Immunofluorescence studies of competition with PaKT showed that human KTAb efficiently bind to the specific $P a K T R$ on the surface of rat-derived and human $P$. carinii organisms.

Conclusions: The results strongly suggest that human $\mathrm{KTAb}$, elicited by a common transphyletic receptor of different pathogenic microorganisms during infection, may play a role in antibody-mediated cross-immunity and, if properly engineered, as functionally equivalent recombinant antibodies they could exert a therapeutic activity against pneumocystosis in vivo.

\section{INTRODUCTION}

Although the development of new therapeutic strategies against pneumocystosis remains a priority, few drugs capable of inhibiting specifically

Address correspondence and reprint requests to: Prof. Luciano Polonelli, Istituto di Microbiologia, Facoltà di Medicina e Chirurgia, Università degli Studi di Parma, Via A. Gramsci 14, 43100 Parma, Italy. Phone: 011-39-521988877; fax: 011-39-521-993620 the growth of Pneumocystis carinii without secondary effects are known (1). During the last few years, the susceptibility of mouse- or rat-derived $P$. carinii to a Pichia anomala candidacidal killer toxin $(\mathrm{PaKT})$ has been demonstrated by using in vitro attachment tests or infectivity assays (2-4). However, the $P a \mathrm{KT}$, which is toxic and antigenic, other than inactive under human physiological conditions, could not be used as a systemic antibiotic $(5,6)$. For this reason, a new strategy to 
control candidiasis or pneumocystosis was developed by using yeast killer toxin-like anti-idiotypic antibodies (KT-IdAb) (6,7). An immunoglobulin Gl (IgGl) monoclonal antibody (MAbKT4) that neutralized in vitro the fungicidal activity of $\mathrm{PaKT}$ was used as an immunogen (idiotypic vaccination) to elicit KT-IdAb capable of inhibiting in vitro the growth of PaKT-sensitive Candida albicans $(8-11)$. KT-IdAb raised in sera and vaginal secretions of mice and rats through parenteral and intravaginal immunization with MAbKT4 were able to confer immunoprotection against systemic or mucosal experimental candidiasis $(9,10)$. Presumably, the paratope of MAbKT4 has regions that are similar to those of the yeast killer toxin receptor ( $P a \mathrm{KTR}$ ) at the surface of sensitive target microbial cells. Thus, KT-IdAb reactive with the MAbKT4 idiotype could also react with the PaKTR at the surface of $C$. albicans or $P$. carinii cells $(2,12)$. The immune system therefore should recognize the PaKTR of infectious microorganisms such as the MAbKT4 idiotype, and consequently, anti-receptor yeast killer toxinlike antibodies (KTAb) should be part of the antibody repertoire of animals experimentally infected with PaKTR-bearing microorganisms or of patients affected by candidiasis. Anti-receptor KTAb have been elicited and detected in animals never immunized with MAbKT4 but repeatedly infected with PaKT-sensitive C. albicans cells (13). Likewise, human natural KTAb prevalently of the IgA isotype were found in the vaginal secretions of patients affected by vaginal candidiasis (13). MAbKT4-affinity chromatography-purified human KTAb proved to be as candidacidal in vitro as those $\mathrm{KT}$-IdAb raised in rat vaginas by idiotypic vaccination with MAbKT4 (10). The antifungal activity of human KTAb was totally neutralized by previous reaction with MAbKT4, confirming the high specificity of their killer effect. Human natural purified KTAb were also capable of transferring passive immunity to naive animals experimentally infected with PaKTRbearing $C$. albicans cells (13). Human natural $\mathrm{KTAb}$ were shown to interact with specific $\mathrm{Pa}$ KTR of $C$. albicans cells by competition experiments with $\mathrm{PaKT}$ in immunofluorescence assays (14).

In this study, the anti- $P$. carinii activity of human natural candidacidal KTAb was evaluated. They were tested for their ability to inhibit the in vitro attachment of rat-derived $P$. carinii to cultured cells as well as the infectivity of the same organism to nude rats. PaKT was used to control the sensitivity of $P$. carinii cells to the killer effect and to test its binding to PaKTR of rat-derived and human $P$. carinii in competition with human KTAb.

\section{MATERIALS AND METHODS}

\section{Production of PaKT}

A standard suspension of $5 \times 10^{8} \mathrm{P}$. anomala ATCC 96603 (formerly defined as UCSC 25F) yeast killer cells, grown for $24 \mathrm{hr}$ at $25^{\circ} \mathrm{C}$ on Sabouraud agar (Diagnostic Pasteur, Paris, France), was inoculated into $100 \mathrm{ml}$ flasks of Sabouraud broth, buffered at $\mathrm{pH} 4.6$. After $48 \mathrm{hr}$ at $25^{\circ} \mathrm{C}$, the yeast suspension was centrifuged and the supernatant containing the PaKT was filtered through $0.22-\mu \mathrm{m}$ filters as previously described (4).

\section{Monoclonal Antibody}

An IgGl monoclonal antibody (MAbKT4), which neutralized the activity of $\mathrm{PaKT}$ against recognized $P a K T$-sensitive strains of $C$. albicans, was produced according to the standard procedures previously described (15). MAbKT4 was produced as ascitic fluid, purified by ammonium sulfate precipitation, dialyzed against Dulbecco's phosphate-buffered saline (PBS), and stored at $-20^{\circ} \mathrm{C}$.

\section{Human Natural Killer Toxin-like Candidacidal Antibodies (KTAb)}

Human natural KTAb were obtained and purified as described recently (13). Briefly, natural $\mathrm{KTAb}$ present in the vaginal secretions of women afflicted by candidal vaginitis were purified by MAbKT4-affinity chromatography and dialyzed against PBS. The candidacidal activity of these purified KTAb was controlled by using a colonyforming unit (CFU) assay against $C$. albicans cells sensitive to the activity of PaKT according to a procedure previously described (13). In this study, candidacidal KTAb from two patients (A and $B$ ) at the approximate concentration of 175 $\mu \mathrm{g} / \mathrm{ml}$ were used separately. Patients A and B corresponded to patient 1 (KTAb of prevalent isotype $M$ ) and patient 8 (KTAb of prevalent isotype G), respectively, as described by Polonelli et al. (13). Vaginal secretions from patients $A$ and $B$ that were not fixed by the MAbKT4 affinity chromatography (KTAb-depleted) were used as negative controls. 


\section{Source and Quantification of $P$. carinii Organisms}

Corticosteroid-treated outbred Wistar rats (Iffa Credo, Lyon, France) were used as the source of the parasite. They were administered dexamethasone (Merck Sharp and Dohme, Qualimed, France) in drinking water $(2 \mathrm{mg} / \mathrm{l})$ for 9-12 weeks. Animals were housed in a conventional room and were given standard food (UAR, France) and water ad libitum. Parasite extraction and quantification were performed as previously described (2).

\section{In Vitro Attachment Assay}

WI38 VAl3 subline 2RA (ECACC 85062512) cells were used as target cell populations. These epithelial lung cells were selected to develop an in vitro assay having similarities with in vivo infection models. WI38 VAl 3 cells were grown in Dulbecco's modified eagle's medium (DMEM; Biowhittaker, Verviers, Belgium) supplemented with $10 \%$ heat-inactivated fetal calf serum (FCS) on glass coverslips placed in the wells of 24-well flat bottom plates (Costar, Brumath, France). When cell monolayer cultures were subconfluent, the medium was removed and $1 \mathrm{ml}$ of a suspension of $4 \times 10^{6}$ parasites in DMEM, $\mathrm{pH}$ 7.2 , was placed in each well. Before being incubated with cultured cells, $P$. carinii organisms were treated for $90 \mathrm{~min}$ at $37^{\circ} \mathrm{C}$ in $500 \mu$ lof the following reagents: DMEM, $\mathrm{pH} 7.2$; KTAb immunopurified from patients $A$ or $B$; vaginal secretions from patients $A$ and $B$ that were not fixed by the MAbKT4 chromatography (KTAb-depleted); and KTAb (from patient B) mixed with MAbKT4. The last two reagents were used to control the specificity of human KTAb activity. The plates were incubated for $24 \mathrm{hr}$ in an atmosphere of $5 \% \mathrm{CO}_{2}$ at $37^{\circ} \mathrm{C}$. After incubation, the coverslips were washed three times with phosphate buffer at $37^{\circ} \mathrm{C}$ to eliminate unattached and dead parasites. Each experiment was performed in triplicate. Attached P. carinii organisms were microscopically detected using methanol-Giemsa and counted by a previously described method $(2,16)$.

\section{Nude Rat Model}

Male and female nude (nu/nu) rats 8 weeks of age were used as recipient animals. These animals came from a colony (Pasteur Institute of Lille, France) previously used in several $P$. carinii transmission experiments which had been found to be free of latent $P$. carinii infection. They were immunosuppressed for 15 days with corticosteroid (dexamethasone; Merck Sharp and Dohme, France) in their drinking water $(1 \mathrm{mg} / \mathrm{l})$. Under corticosteroid administration, the parasite number showed low interindividual or intersexual variation. Nude rats were anesthetized with a drug cocktail given intraperitoneally (17). They were inoculated by the intratracheal route with $10 \times 10^{7}$ parasites $/ 100 \mu \mathrm{l}$ of medium along with $0.3 \mathrm{ml}$ of air. In these inocula, the cysts' percentage by comparison with trophozoites was $4-7 \%$. The tracheal wound was closed with nonresorbable sutures (3/8 Seracap, Serquighy, France). All manipulations were carried out under sterile conditions and the hooded rat cages were placed in sterile boxes maintained under sterile air-flow (Esi Flufrance, Wissous, France). The cages, food, water, and bedding were sterilized before being used.

\section{Evaluation of $P$. carinii Infectivity in Nude Rats}

The experimental animal model of nude rats was used at first to control that $P$. carinii infectivity was affected by the antibiotic activity of PaKT. For this evaluation, five independent experiments were performed with four groups of four animals each. Samples of freshly extracted $P$. carinii organisms were incubated for $90 \mathrm{~min}$ at $25^{\circ} \mathrm{C}$ in $500 \mu \mathrm{l}$ of the following reagents before being inoculated into the animals: DMEM, $\mathrm{pH}$ 7.2; $\mathrm{PaKT}, \mathrm{pH} 4.6$ (active killer toxin); PaKT, pH 7.2 (inactive killer toxin); and $P a K T$ that had been neutralized by mixing with mAbKT4 for 90 $\min$ at $25^{\circ} \mathrm{C}$. Another control group was represented by rats inoculated with parasite-free DMEM.

For the evaluation of human natural KTAb antimicrobial activity, before being inoculated in animals, samples of freshly extracted parasites were incubated for $90 \mathrm{~min}$ at $37^{\circ} \mathrm{C}$ in $500 \mu \mathrm{l}$ of the following preparations in PBS ( $\mathrm{pH}$ 7.2): KTAb-depleted vaginal secretions from patients $\mathrm{A}$ and B; KTAb (patient A); and KTAb (patient A) neutralized by previous mixing with MAbKT4.

The treated parasite samples were then centrifuged before being resuspended in complete fresh DMEM, pH 7.2, and inoculated to immunosuppressed nude rats (4 rats/group) as described above.

All rats were given dexamethasone in their drinking water for 40 days. They were then sac- 
rificed for $P$. carinii organism extraction and count.

\section{Immunofluorescence Assay}

The immunofluorescence studies were carried out with rat-derived and human $P$. carinii organisms obtained from experimental animals and bronchial washings of patients affected by pneumocystosis by using biotinylated $P a K T$ in competition with human natural KTAb according to a procedure previously described $(18,19)$. Briefly, concentrated $P a K T(8 \mathrm{mg} / \mathrm{ml})$ was dialyzed against $500 \mathrm{ml}$ of labeling buffer $\left(0.1 \mathrm{M} \mathrm{NaHCO}_{3}\right.$, $0.1 \mathrm{M} \mathrm{NaCl}$ adjusted to $\mathrm{pH} 7.4$ with concentrated $\mathrm{HCl}$ ) at $4^{\circ} \mathrm{C}$ with three changes over 2 days. After dialysis, $30 \mu \mathrm{l}$ of $10 \mathrm{mg} / \mathrm{ml}$ biotin (Calbiochem, La Jolla, CA) in dimethyl sulfoxide (Sigma Chemical Co., St. Louis, MO) were added to each milligram of $P a K T$. The mixture was incubated for $3 \mathrm{hr}$ at room temperature in the dark and at slow agitation. The unbound biotin was removed by dialysis against $500 \mathrm{ml}$ of PBS at $4^{\circ} \mathrm{C}$ with three changes over 2 days. The biotinylated $\mathrm{PaKT}$ was maintained at $4^{\circ} \mathrm{C}$ in the dark until used. Twenty microliters of the biotinylated PaKT (diluted 1:2 in PBS) was added to each well of an immunofluorescence slide containing fixed $P$. carinii organisms of rat and human origin and maintained for $1 \mathrm{hr}$ at $37^{\circ} \mathrm{C}$ in a humid chamber. The slide was successively washed three times (10 min each) with shaking in PBS. The slide was allowed to dry at room temperature and $20 \mu \mathrm{l}$ of streptavidin-fluorescein (Amersham International, Little Chalfont, England) diluted 1:100 in Evans blue was added to each well and allowed to react for 20 min under the same conditions as above. The slide was then rinsed with distilled water and mounted with a coverslip using a mounting fluid (Syva Microtrak, Palo Alto, CA). The slide was observed with a fluorescence microscope (Zeiss Axiophot, Jena, Germany). As a negative control, the immunofluorescence assay was carried out by using, in the same procedure, PBS in place of biotinylated PaKT. For competition experiments, the same procedure was carried out by using biotinylated $P a K T$ diluted $1: 2$ in human natural KTAb.

\section{Statistical Analysis}

Results were expressed as mean values \pm standard deviation. Differences in parasite numbers



FIG. 1. Inhibitory effect of human natural yeast killer toxin-like antibodies (KTAb) on the attachment of rat-derived $P$. carinii to WI38 VA13 cells

Before the attachment assay, the parasites were preincubated in different reagents: DMEM, pH 7.2 (1); KTAb immunopurified from patient A (2); KTAb immunopurified from patient B (3); KTAb-depleted vaginal secretions (4); and KTAb (from patient B) neutralized by MAbKT4 (5). ${ }^{*} p<0.001$, the parasite number compared with that of parasites preincubated in KTAb-depleted vaginal secretions.

were evaluated by using the Student $t$-test $(p<$ 0.05 was considered significant).

\section{RESULTS}

\section{Effect of Human KTAb on P. carinii in Vitro Attachment}

Human natural KTAb immunopurified from the vaginal secretions of patients $A$ and $B$ induced a significant and similar inhibition of rat-derived $P$. carinii attachment to WI38 VA13 cells $(81 \pm$ $13.3 \times 10^{4}$ and $78 \pm 20.1 \times 10^{4}$ attached parasites, respectively). In contrast, this inhibitory effect was not observed when parasites were treated either with DMEM (pH 7.2) or KTAbdepleted vaginal secretions from the same patients used as controls $\left(190 \pm 23.6 \times 10^{4}\right.$ and $176 \pm 28.5 \times 10^{4}$ attached parasites, respectively) (Fig. 1). The inhibition level obtained with KTAb (54\% for patient A and $55.5 \%$ for patient B) was evaluated in comparison to that obtained with KTAb-depleted vaginal secretions. 


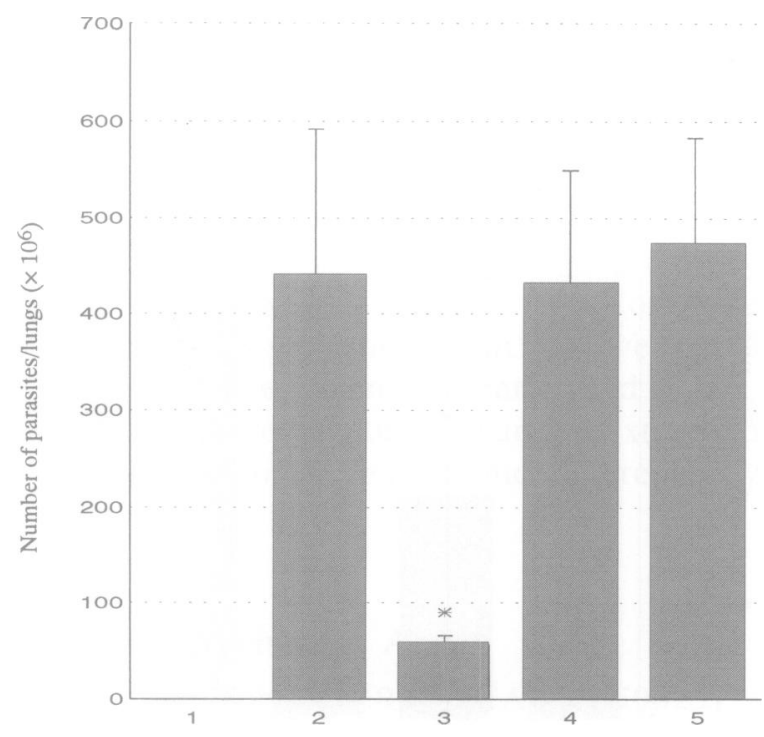

FIG. 2. Inhibitory effect of PaKT on the infectivity of rat-derived $P$. carinii to nude rats

In this experiment, a control group consisted of animals inoculated with DMEM without parasites (1). Parasites were incubated for $90 \mathrm{~min}$ at $25^{\circ} \mathrm{C}$ in the following reagents before being inoculated in animals: DMEM, pH 7.2 (2); PaKT, pH 4.6 (active killer toxin) (3); $P a \mathrm{KT}$, pH 7.2 (inactive killer toxin) (4); and $\mathrm{PaKT}$ neutralized for $90 \mathrm{~min}$ at $25^{\circ} \mathrm{C}$ by MAbKT4 (5). * $p<0.001$, the parasite number compared with that of parasites preincubated in DMEM, pH 7.2.

Moreover, when rat-derived $P$. carinii organisms were treated with KTAb from patient B neutralized by MAbKT4, the number of parasites attached to WI38 VA13 cells was similar to that obtained in the two controls $\left(162 \pm 28.4 \times 10^{4}\right)$.

\section{Effect of PaKT on P. carinii Infectivity in Nude Rats}

Five experiments were carried out consecutively within 1 year. The corticosteroid-treated nude rats were nonlatently infected by $P$. carinii because animals not inoculated with parasites did not develop pneumocystosis (control without parasite). Figure 2 shows that rat-derived parasites preincubated in DMEM, $\mathrm{pH} 7.2$, induced severe pneumocystosis in nude rats (439 \pm $155 \times 10^{6}$ parasites/animal). The number of parasites in rats inoculated with inocula treated with active $P a K T$ ( $\mathrm{pH}$ 4.6) was significantly lower $\left(57 \pm 10 \times 10^{6}\right.$ parasites/animal) than that recorded in animals inoculated with parasites treated with either inactive $P a K T \quad(p H$ 7.2) $\left(431 \pm 120 \times 10^{6}\right.$ parasites/animal) or parasites treated with active $P a K T$ neutralized by MAbKT4 $\left(472 \pm 113 \times 10^{6}\right.$ parasites/animal $)$. An effect due to the acidity of active PaKT was also excluded on the basis of previous data showing that parasites preincubated in DMEM, $\mathrm{pH} 4.6$, remained as viable and infective as parasites preincubated in DMEM, pH 7.2 (2). Histological examination showed that lungs of nude rats infected with parasites pretreated with inactive PaKT (pH 7.2) or active PaKT (pH 4.6) neutralized by MAbKT4 were characterized by the presence of an eosinophilic exsudate typical of pneumocystosis which was absent in lungs of animals infected with parasites that had been pretreated with PaKT, pH 4.6 (data not shown).

\section{Effect of KTAb on $P$. carinii Infectivity in Nude Rats}

Rat-derived parasites preincubated in KTAb-depleted vaginal secretions from patients $A$ and $B$ induced severe pneumocystosis in nude rats (246 $\pm 45 \times 10^{6}$ parasites/animal). On the contrary, when $P$. carinii organisms were preincubated in purified human KTAb from the same patients, their infectivity was inhibited $(63.5 \pm$ $17 \times 10^{6}$ parasites/animal). In comparison with the appropriate control, the percentage of inhibition was $74.2 \%$ when the $P$. carinii organisms were pretreated with KTAb. MAbKT4 proved to neutralize the inhibitory activity of KTAb (from patient $A$ ) as attested by the recorded number of parasites $\left(206 \pm 6.8 \times 10^{6}\right.$ parasites/animal), which was comparable to that obtained in rats inoculated with parasites pretreated with KTAbdepleted vaginal secretions (Fig. 3).

\section{Visualization of PaKTR in $P$. carinii}

Immunofluorescence studies carried out by using biotinylated $\mathrm{PaKT}$ permitted visualization of the putative KTR in rat-derived and human $P$. carinii organisms (Fig. 4). The immunofluorescence reactivity was lost by the competition of human natural KTAb against $P a \mathrm{KT}$ for binding to the surface of $P$. carinii organisms of both animal and human origin.

\section{DISCUSSION}

$P$. carinii is an opportunistic microorganism recently reclassified in the kingdom Fungi on the basis of nucleic acid analysis $(20-23)$. P. carinii pneumonia became an increasingly important 


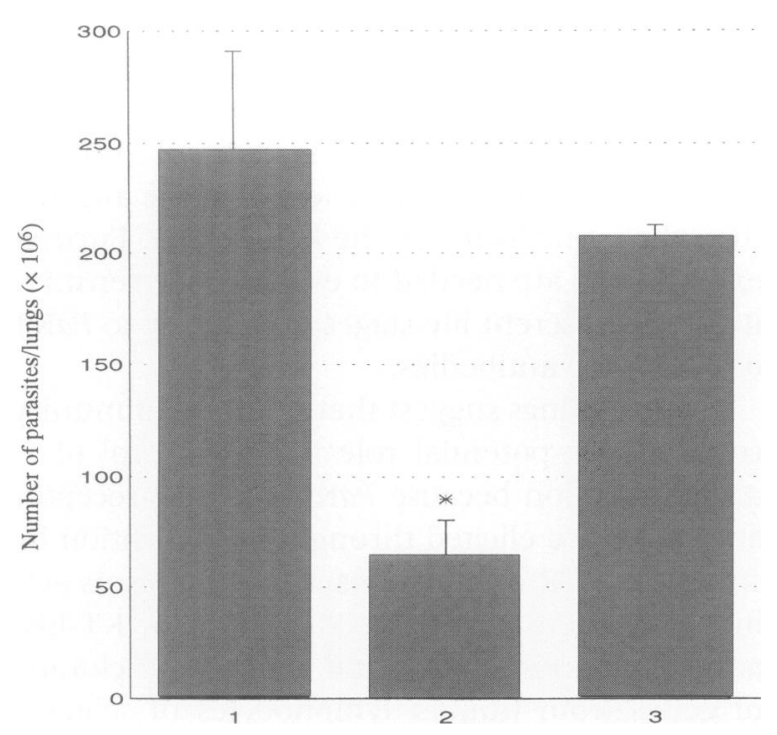

FIG. 3. Inhibitory effect of the human natural yeast killer toxin-like antibodies (KTAb) on the infectivity of rat-derived $P$. carinii to nude rats

The preincubation media were: KTAb-depleted vaginal secretions (1); KTAb from patient A (2); and KTAb (from patient A) neutralized by MAbKT4 (3). ${ }^{*} p<0.001$, the parasite number compared with that of parasites preincubated in KTAb-depleted vaginal secretions.

problem in terms of morbidity and mortality among immunocompromised patients, such as those receiving transplantations and immunosuppressive drugs. The advent of AIDS brought about an explosive increase in its prevalence. $P$. carinii became the most common life-threatening opportunistic pathogen in HIVinfected people (24). In immunocompetent hosts, P. carinii infection generally stimulates cell-mediated and antibody immunity. There is strong evidence that both of these types of immune responses are important for the control of infections. The precise role of humoral immunity, however, remains to be clarified (25-27). An improved definition of the mechanisms of host immune response would contribute to the development of new vaccination strategies for the control of $P$. carinii infections. The identification of new cellular targets would also constitute the basis for the development of new drugs against pneumocystosis. Interestingly, new lipopeptidic cyclic antifungal compounds that inhibit the synthesis of $\beta$ 1-3 glucan, such as echinocandin and papulocandin, have proven to be highly effective in vivo against $P$. carinii (28). In the cell wall of $P$. carinii,

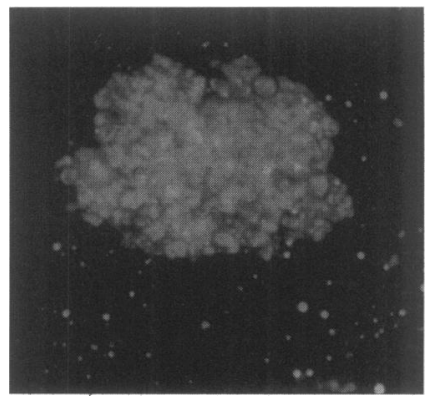

FIG. 4. Immunofluorescence visualization of putative yeast killer toxin cell wall receptors on human P. carinii by biotinylated PaKT

the presence of yeast glucan has been revealed (29). This represents one the major constituents of cell wall receptors for some yeast killer toxins such as that of $P$. anomala, which displays a large spectrum of antimicrobial activity against many pathogenic microorganisms, including $C$. albicans, a common opportunistic pathogen in HIV patients (30-32). In previous studies, we demonstrated that a candidacidal $P$. anomala killer toxin $(P a K T)$ was also able to inhibit the in vitro attachment of rat-derived $P$. carinii (2) or its in vivo infectivity (3). These antimicrobial activities have proven to be highly specific because they can be abolished by prior adsorption of $P a K T$ with a neutralizing monoclonal antibody (MAbKT4) that also allows the immunofluorescence detection of specific PaKTR on the cell wall of $P$. carinii organisms treated with PaKT (2). MAbKT4 has been extensively used to vaccinate mice and rats by parenteral or mucosal administration against experimental intravenous and intravaginal infections with PaKT-sensitive $C$. albicans cells (idiotypic vaccination) $(9,10)$. Immunoprotection was related to the elicitation in the serum or vaginal secretions of vaccinated animals of high titers of PaKT-like antidiotypic antibodies (KT$\mathrm{IdAb})$ that were shown to interact with putative C. albicans PaKTR and to kill in vitro the C. albicans cells used for experimental challenge. They also conferred passive immunity when transferred to unvaccinated animals, thus confirming that they represented the internal image of $\mathrm{PaKT}$. Inherent studies based on the speculation that the dual steric homology between $P a K T$ and KT-IdAb should conversely imply one between the paratope of MAbKT4 used as a vaccine in idiotypic vaccination and the PaKTR of sensitive C. albicans cells, led to the detection of antireceptor $\mathrm{PaKT}$-like candidacidal antibodies (KTAb) in the vaginal secretions of rats experimentally in- 
fected with $C$. albicans cells and in women with vaginal candidiasis (13). The goal of this study was to verify if such natural candidacidal antibodies representing the internal image of $\mathrm{PaKT}$ could also display activity against recognized $P a K T$-sensitive $P$. carinii organisms, even though the study was not designed to draw definitive conclusions about the relationship of antibody concentration to killer effect. We have shown that human natural candidacidal KTAb were able to inhibit the in vitro attachment of rat-derived $P$. carinii organisms to epithelial lung cells as well as parasite infectivity in the nude rat model (16). Data obtained from the in vitro attachment assay showed that $55.5 \%$ of the human natural KTAb-treated parasites were not attached to WI38 VAl 3 cells. The same effect had been observed by using PaKT-treated parasites in other cell line systems $(2,3)$. Human natural KTAb were shown to display an inhibitory activity comparable to that of PaKT (16). Data obtained with the nude rat model confirmed this finding by showing inhibition of the infectivity of $P$. carinii organisms treated with KTAb as well as with PaKT. Moreover, when human KTAb were adsorbed with mAbKT4, the inhibitory effect on the attachment of parasite and infectivity was significantly neutralized $(p<0.001)$. These data suggest that $\mathrm{KTAb}$, representing the internal image of $P a K T$, were able to interact with the idiotype of PaKT-neutralizing MAbKT4, thus attesting to the specificity of their antibiotic activity.

As marked differences related to host species were reported among $P$. carinii strains $(33,34)$, it could be argued that the results are only applicable to rat-derived $P$. carinii isolates. However, we have previously shown that $\mathrm{PaKT}$ is also active against mouse-derived $P$. carinii (4). Moreover, $P a \mathrm{KT}$ or KTAb are equally active against $C$. albicans, which suggests that the killer activity of these compounds is dependent on transphyletic receptors (14). The putative PaKTR on C. albicans and $P$. carinii, which were cytochemically located by using polyclonal rabbit KT-IdAb or PaKT and MAbKT4 with indirect immunofluorescence assays $(2,12)$, were also visualized either in ratderived or human $P$. carinii organisms by using biotinylated $\mathrm{PaKT}$ in the direct immunofluorescence procedure. The competition exerted by $\mathrm{KTAb}$ to PaKT, particularly in the human clinical samples, proved that despite the animal origin of parasite, these antibodies bind to the same $\mathrm{Pa}$ KTR of $P$. carinii as PaKT.
Although our experiments demonstrated the ability of KTAb to inhibit rat-derived parasite in vitro attachment and in vivo infectivity to the nude rat, these observations did not indicate whether trophozoites were less or more affected than the cystic forms by the killer effect. Further experiments are needed to evaluate the sensitivity of the different life stages of $P$. carinii to $P a \mathrm{KT}$ or PaKT-like antibodies.

Our findings suggest that humoral immunity could play a potential role in the control of $P$. carinii infection because PaKT-like anti-receptor antibodies are elicited through immunization by specific PaKTR of infectious microorganisms acting as antigens (14). The validation of KT-like antibodies, moreover, could lead to the cloning of genes from human lymphocytes of selected donors that encode for KTAb-variable regions, thus obtaining Fab characterized by antibiotic activity that could be most properly used as immunotherapeutants in the treatment of pneumocystosis. The potential therapeutic effect of murine yeast killer toxin-like monoclonal and single-chain recombinant antibodies previously described in an experimental model of candidal vaginitis strongly supports this expectation $(18,19)$.

Further studies using properly engineered MAbKT4 idiotype-like vaccines will be required to extend the prophylactic value of idiotypic vaccination against experimental pneumocystosis to ascertain if it could be used in individuals at risk of developing the disease.

\section{ACKNOWLEDGMENTS}

The authors thank Ms. Chantal Mullet from U42 INSERM (Villeneuve d'Ascq) for the numeration of parasites, and Mr. Emile Fleurbaix and Pierre Billiaut from the Institut Pasteur (Lille) for providing the nude rats. This work was developed as part of a European BIOMED-1 Concerted Action entitled "Pneumocystis and Pneumocystosis. Impact of the Biodiversity of Pneumocystis carinii on Epidemiology, Pathology, Diagnosis, Monitoring and Prevention of Pneumocystosis. New Therapeutic Approaches" (BMHI-CT94-1118). This project was also supported by the Agence Nationale de Recherches sur le SIDA (ANRS). N.S. was awarded a grant from ANRS (94635). L.P. and S.C. were supported by the Ministero della Sanità-Istituto Superiore di Sanità, IX Progetto AIDS Roma, Italy (9404/28). 


\section{REFERENCES}

1. Sattler FR, Jelliffe RW. (1994) Pharmacokinetic and pharmacodynamic considerations for drug dosing in the treatment of Pneumocystis carinii pneumonia. In: Walzer PD (ed). Pneumocystis carinii Pneumonia, 2nd ed., Vol. 69. Marcel Dekker, New York, pp. 467485.

2. Aliouat EM, Cailliez JC, Séguy $\mathrm{N}$, et al. (1993) Inhibitory effect of a yeast killer toxin to the in vitro Pneumocystis carinii attachment. Serodiagn. Immunother. Infect. Dis. 5: 102-106.

3. Séguy N, Aliouat EM, Dei-Cas E, Polonelli L, Camus D, Cailliez JC. (1994) Susceptibility of Pneumocystis carinii to a Pichia anomala killer toxin. J. Eukaryot. Microbiol. 41: 109S.

4. Séguy N, Cailliez JC, Polonelli L, Dei-Cas E, Camus D. (1996) Inhibitory effect of a Pichia anomala killer toxin on Pneumocystis carinii infectivity to the SCID mouse. Parasitol. Res. 82: 114-116.

5. Conti S, Cantelli C, Gerloni M, et al. (1996) Killer factor interference in mixed opportunistic yeast cultures. Mycopathologia 135: 1-8.

6. Polonelli L, Conti S, Gerloni M, Magliani W, Chezzi C, Morace G. (1991) Interfaces of the yeast killer phenomenon. Crit. Rev. Microbiol. 8: 47-87.

7. Cailliez JC, Séguy N, Aliouat EM, Polonelli L, Camus D, Dei-Cas E. (1994) The yeast killer phenomenon: A hypothetical way to control Pneumocystis carinii pneumonia. Med. Hypotheses 43: 167-171.

8. Polonelli L, Morace G. (1988) Yeast killer toxin-like anti-idiotypic antibodies. J. Clin. Microbiol. 26: 602-604.

9. Polonelli L, Lorenzini F, DeBernardis F, et al. (1993) Idiotypic vaccination: Immunoprotection mediated by anti-idiotypic antibodies with antibiotic activity. Scand. J. Immunol. 37: 105-110.

10. Polonelli L, De Bernardis F, Conti S, et al. (1994) Idiotypic intravaginal vaccination to protect against candidal vaginitis by secretory, yeast killer toxin-like anti-idiotypic antibodies. J. Immunol. 152: 3175-3182.

11. Polonelli L, Conti S, Gerloni M, et al. (1991) 'Antibiobodies': Antibiotic-like antiidiotypic antibodies. J. Med. Vet. Mycol. 29: 235-242.

12. Polonelli L, Fanti F, Conti S, et al. (1990) Detection by immunofluorescent anti-idiotypic antibodies of yeast killer toxin cell wall receptors of Candida albicans. J. Immunol. Methods 132: 205-209.
13. Polonelli L, De Bernardis F, Conti S, et al. (1996) Human natural yeast killer toxin-like candidacidal antibodies. J. Immunol. 156: 1880-1885.

14. Cassone A, Conti S, De Bernardis F, Polonelli L. (1997) Antibodies, killer toxins and antifungal immunoprotection: A lesson from nature? Immunol. Today 18: 164-169.

15. Polonelli L, Morace G. (1987) Production and characterization of yeast killer toxin monoclonal antibodies. J. Clin. Microbiol. 25: 460-462.

16. Séguy N, Polonelli L, Conti S, Dei-Cas E, Camus D, Cailliez JC. (1996) In vitro decrease of rat-derived Pneumocystis carinii attachment induced by human natural yeast killer toxinlike antiidiotypic candidacidal antibodies. $J$. Eukaryot. Microbiol. 43: 27S.

17. Aliouat EM, Dei-Cas E, Billaut P, Dujardin L, Camus D. (1995) Pneumocystis carinii organisms from in vitro culture are highly infectious to the nude rat. Parasitol. Res. 81: 82-85.

18. Magliani W, Conti S, De Bernardis F, et al. (1997) Therapeutic potential of antiidiotypic single chain antibodies with yeast killer toxin activity. Nat. Biotechnol. 15: 155-158.

19. Polonelli L, Séguy N, Conti S, et al. (1997) Monoclonal yeast killer toxin-like candidacidal antiidiotypic antibodies. Clin. Diagn. Lab. Immunol. 4: 142-146.

20. Cailliez JC, Séguy N, Denis CM, et al. (1996) Pneumocystis carinii: An atypical fungal microorganism. J. Med. Vet. Mycol. 34: 227-239.

21. Edman JC, Sogin ML. (1994) Molecular phylogenic of Pneumocystis carinii. In: Pneumocystis carinii Pneumonia, 2nd ed., New York, Marcel Dekker, Vol. 69. pp. 91-106.

22. Stringer JR, Edman JC, Cushion MT, Richards FF, Watanabe J. (1992) The fungal nature of Pneumocystis carinii. J. Med. Vet. Mycol. 30: 271-278.

23. Wakefield AE, Peters SE, Banerji S, Bridge PD, Hopkin JM. (1992) Pneumocystis carinii shows DNA homology with the Ustomycetous Red yeast fungi. Mol. Microbiol. 6: 19031911.

24. Walzer PD. (1991) Pneumocystis carinii-New clinical spectrum? New Engl. J. Med. 324: 263-265.

25. Beck JM, Liggitt HD, Brunette EN, Fuchs HJ, Shellito JE, Debs RJ. (1991) Reduction in intensity of Pneumocystis carinii pneumonia in mice by aerosol administration of gamma interferon. Infect. Immunol. 59: 3859-3862.

26. Ezekowitz RAB, Williams DJ, Koziel H, et al. (1991) Uptake of Pneumocystis carinii medi- 
ated by the macrophage mannose receptor. Nature 351: 155-158.

27. Su TH, Martin WJ. (1994) Pathogenesis and host response in Pneumocystis carinii pneumonia. Annu. Rev. Med. 45: 261-272.

28. Schmatz D, Romancheck M, Pittarelli L, et al. (1990) Treatment of Pneumocystis carinii with 1,3-glucan synthesis inhibitors. Proc. Natl. Acad. Sci. USA 87: 5950-5954.

29. Matsumoto Y, Matsuda S, Tegashi T. (1989) Yeast glucan in the cyst wall of Pneumocystis carinii. J. Protozool. 36: 21S-24S.

30. Sawant AD, Ahearn DG. (1990) Involvement of a cell wall receptor in the mode of action of an anti-Candida toxin of Pichia anomala. Antmicrob. Agents Chemother. 34: 1331-1335.
31. Polonelli L, Morace G. (1986) Reevaluation of the yeast killer phenomenon. J. Clin. Microbiol. 24: 866-869.

32. Magliani W, Conti S, Gerloni M, Bertolotti D, Polonelli L. (1997) Yeast killer systems. Clin. Microbiol. Rev. 10: 369-400.

33. Kovacs JA, Halpern JL, Lungren B, Swan JC, Parrillo JE, Masur H. (1989) Monoclonal antibodies to Pneumocystis carinii: Identification of specific antigens and characterization of antigenic differences between rat and human isolates. J. Infect. Dis. 159: 60-70.

34. Mazars E, Ödberg-Ferragut C, Dei-Cas E, et al. (1995) Polymorphism of the thymidylate synthase gene of Pneumocystis carinii from different host species. J. Eukaryot. Microbiol. 42: $26-32$.

Communicated by A. Martinez-Palomo. Accepted June 18, 1997. 